\title{
LA-UR-16-26352
}

Approved for public release; distribution is unlimited.

Title:

Thermal Activation of Dislocations in Large Scale Obstacle Bypass

Author(s):

Martinez Saez, Enrique

Sobie, Cameron

Wen, Wei

Capolungo, Laurent

Patra, Anirban

McDowell, David L.

Tome, Carlos

Intended for: Report

Issued:

2016-08-18 
Disclaimer:

Los Alamos National Laboratory, an affirmative action/equal opportunity employer, is operated by the Los Alamos National Security, LLC for the National Nuclear Security Administration of the U.S. Department of Energy under contract DE-AC52-06NA25396. By approving this article, the publisher recognizes that the U.S. Government retains nonexclusive, royalty-free license to publish or reproduce the published form of this contribution, or to allow others to do so, for U.S. Government purposes. Los Alamos National Laboratory requests that the publisher identify this article as work performed under the auspices of the U.S. Department of Energy. Los Alamos National Laboratory strongly supports academic freedom and a researcher's right to publish; as an institution, however, the Laboratory does not endorse the viewpoint of a publication or guarantee its technical correctness. 


\section{Thermal Activation of Dislocations in Large Scale Obstacle Bypass}

\section{NEAMS Milestones report 08/01/2016}

\section{Enrique Martinez Saez, Cameron Sobie, Wei Wen, Laurent Capolungo, Anirban Patra, David McDowell and Carlo Tomé.}

Irradiation-created defects diffuse and agglomerate presenting obstacles for the dislocation motion (both glide and climb). This effect leads to hardening and loss of toughness, which might drive the system to failure (see Fig. 1).

Atomistic calculations have been extensively used to describe the basic mechanisms in the interaction of dislocations with defects depending on their character, their size and the temperature. However, their limited size and simulated time precludes them from obtaining statistically representative parameters. On the other hand, continuum approaches like crystal plasticity have been employed to describe the response of a polycrystal to external solicitations. These continuum models are traditionally fitted to experimental values, and thus, their predictive capabilities are limited. In most of the

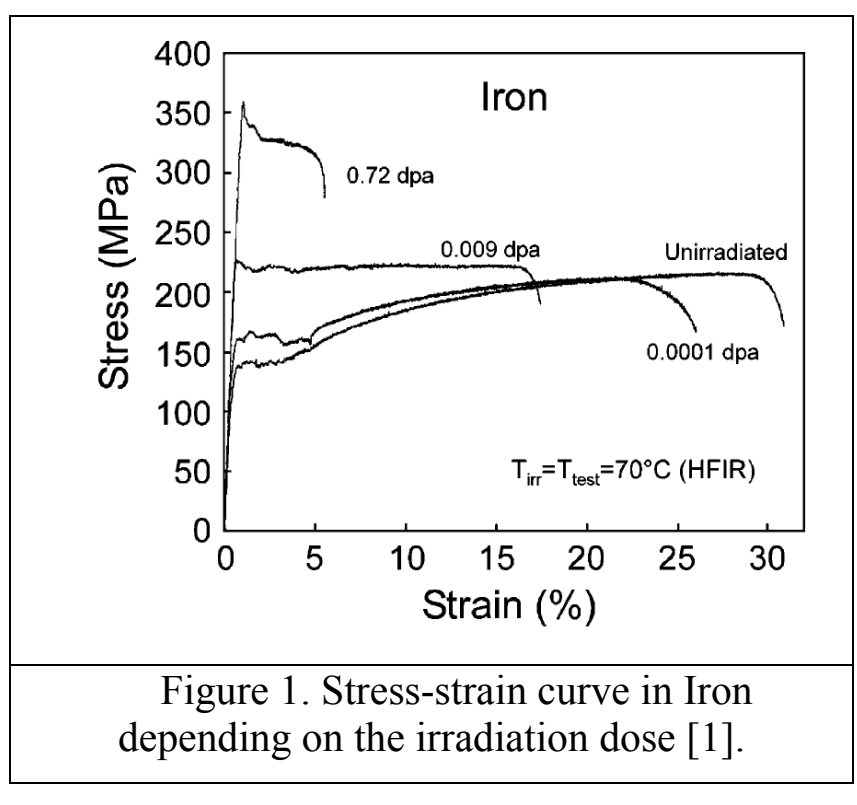
crystal plasticity models, dislocation densities on the different slip systems are the internal variables. Strain hardening is defined by the increase of the critical resolved shear stress, $\tau_{a}^{\alpha}$ with the internal variables in slip system $\alpha$. Such a relationship is introduced through the Mecking and Kocks equation $\tau_{\mathrm{a}}^{\alpha}=\mu \mathrm{b} \sqrt{\sum_{\beta=1}^{\mathrm{N}} \mathrm{a}_{\alpha \beta} \beta^{\beta}}$ where $\mu$ is the shear modulus, $b$ is the Burgers vector, $a_{\alpha \beta}$ is the so called hardening matrix and $\rho^{\beta}$ is the forest dislocation density in system $\beta$. For true predictive capability it is critical that the physics of the extremely correlated dislocation interaction processes be more faithfully described in the CP models. Another parameter of importance in CP simulations is the mean velocity of mobile dislocations, $v^{s}$. This velocity enters the Orowan equation to give the shear rate at a sub-material point:

$$
\dot{\gamma}^{s}=\rho_{m}^{s} b v^{s} \cdot \operatorname{sign}\left(\tau^{s}\right) ; \text { with } v^{s}=\frac{L^{s}}{t_{t}^{s}+t_{w}^{s}}
$$


where $\rho_{m}^{s}$ is the mobile dislocation density in system $s, b$ is the magnitude of the Burgers vector, $L^{S}$ represents the mean distance between obstacles, $t_{t}^{S}$ is the mean time for a dislocation to travel between obstacles and $t_{w}^{s}$ is the average time a dislocation spends waiting at an obstacle ${ }^{2-4}$.

Discrete Dislocation Dynamics (DDD) presents itself as an ideal model to obtain the parameters needed in crystal plasticity methodologies. 3D DDD is a direct approach that simulates the aggregate behavior of large dislocation ensembles at the mesoscale by decomposing dislocation lines of arbitrary curvature and character into piecewise segments $^{5-9}$. Typical length and time scales of current 3D DDD simulations are on the order of microns and seconds offering important insights that help answer fundamental questions in crystal plasticity such as the origin of the complex dislocation patterns that emerge during plastic deformation and the relationship between microstructure, loading conditions and the mechanical strength of the crystal ${ }^{10,11}$. 3D DDD is well adapted to identify the hardening coefficients involved in the Mecking-Kocks equation by simulating the interaction of slip systems $\alpha$ and $\beta$ and measuring the shear stress applied on system $\alpha$ needed to force it to cross the population of dislocations on forest system $\beta$ of density $\rho^{\beta}[12]$.

During this project we have developed a novel DDD methodology that couples traditional approaches with the nudged-elastic band (NEB) method with the main goal of computing the minimum energy path between obstacles in a computationally efficient way that will result in statistically representative values. Moreover, we have also calculated the vibrational frequencies of the dislocations at the equilibrium positions and at the saddle points, that relying on a harmonic transition state theory description of the process gives us access to the rate for the dislocation to overcome the obstacle.

$$
\Gamma_{T S T}=v \cdot \exp \left(\frac{-\Delta E}{k T}\right)
$$

The inverse of this rate is the waiting time that the dislocation has to spend on average before a thermal fluctuation takes it to the other side of the obstacle, which is the waiting time needed in CP calculations.

This development has led to three manuscripts, where the methodology is described in detail. The three of them are currently under review, two on the Journal of Mechanics and Physics of Solids and the third one in Acta Materialia. These manuscripts are attached to this milestone report.

\section{References}

[1] M. Eldrup, B.N. Singh, S.J. Zinkle, T.S. Byun, K. Farrell, Dose dependence of defect accumulation in neutron irradiated copper and iron, Journal of Nuclear Materials 307-311 (2002) 912-917

[2] R. Clifton, On the analysis of elastic/visco-plastic waves of finite uniaxial strain, J. Burke, V. Weiss (Eds.), Shock Waves and the Mechanical Properties of Solids, Syracuse University Press, Syracuse, NY (1971), 73-116.

[3] J.T. Lloyd, J.D. Clayton, , R.A. Austin, , D.L. McDowell, Plane wave simulation of e lastic-viscoplastic single crystals, 69 (2014) 14-32. 
[4] R.A. Austin, D.L. McDowell, A dislocation-based constitutive model for viscoplastic deformation of FCC metals at very high strain rates, International Journal of Plasticity, 27 (2011), 1-24.

[5] Ghoniem, N. M.; Amodeo, R. J. Solid State Phenom. 1988, 3\&4, 377.

[6] Kubin, L.P., Canova, G., Conat, M., Devincre, B., Pontikis, V., Brechet, Y., 1992. Solid State Phenom. 23 \& 24, 455.

[7] Kubin, L.P., Devincre, B., Tang, M., 1998. J. Comput. Aided Mater. Des. 5, 31.

[8] Madec, R., Devincre, B., Kubin, L., Hoc, T. and Rodney, D., Science 301 (2003) 1879.

[9] Bulatov, V. V., Hsiung, L. L. et al., Nature 440 (2006) 1174.

[10] Bulatov, V. V. and Cai, W. "Computer Simulations of Dislocations", Oxford University Press.

[11] Arsenlis, A. et al., Modelling Simul. Mater. Sci. Eng. 15 (2007) 553-595

[12] Fivel M., Etudes numériques à différentes échelles de la déformation plastique des monocristaux de structure cfc, Doctoral thesis, Institute National Polytechnique de Grenoble, 1997. 